PROCEEDINGS OF THE

AMERICAN MATHEMATICAL SOCIETY

Volume 132, Number 10, Pages 2983-2988

S 0002-9939(04)07430-

Article electronically published on May 12, 2004

\title{
SOME INTEGRALS IN RAMANUJAN'S LOST NOTEBOOK
}

\author{
BRUCE C. BERNDT
}

(Communicated by Jonathan M. Borwein)

\begin{abstract}
On page 199 in his lost notebook, Ramanujan recorded three unusual integral formulas. Two of his assertions are correct, and proofs are given here. His third claim is incorrect, but it is true in a limiting form. The integrals arise from a claim in Ramanujan's ordinary notebooks on the equality of certain integrals and series in which the integrand and the summands are themselves the same function.
\end{abstract}

\section{INTRODUCTION}

In Chapter 13 of his second notebook [9, 3, pp. 226-227], Ramanujan briefly examined the problem of finding functions $f$ such that

$$
\int_{-\infty}^{\infty} f(x) d x=\sum_{n=-\infty}^{\infty} f(n)
$$

In particular, he incorrectly asserted that

$$
\int_{-\infty}^{\infty} \frac{a^{x}}{\Gamma(x+1)} d x=e^{a}
$$

If (11.2) were correct, then (1.2) would provide an example of (1.1), since $1 / \Gamma(n+1)=0$ when $n$ is a negative integer. Authors examining instances of (1.1) include R. P. Boas and H. Pollard [4, P. J. Forrester [5], and K. S. Krishnan 7].

Page 199 in Ramanujan's lost notebook is devoted to three integral formulas, which can be considered as attempts to give corrected versions of (1.2). Two of them are correct, but the remaining one is not, although it is true in certain cases. The purpose of this brief note is to provide proofs of the two correct evaluations and to discuss the remaining claim, which is quite interesting despite its being incorrect in general. We now record the three entries on page 199 of the lost notebook [10].

Theorem 1.1. If $a>0$ and $k \geq 0$, then

$$
\int_{-k}^{\infty} \frac{a^{x}}{\Gamma(x+1)} d x+\int_{0}^{\infty} \frac{e^{-a x} x^{k-1}}{\pi^{2}+\log ^{2} x}\left(\cos \pi k-\frac{1}{\pi} \sin \pi k \log x\right) d x=e^{a} .
$$

Received by the editors February 13, 2003.

2000 Mathematics Subject Classification. Primary 33B15; Secondary 40A10.

The author's research was partially supported by grant MDA904-00-1-0015 from the National Security Agency. 
Theorem 1.2. If $a>0$ and $k \geq 0$, then

$$
\int_{-k}^{\infty} \frac{a^{x}}{\Gamma(x+1)} d x+\frac{1}{2 \pi} \int_{0}^{\infty}\left\{\frac{e^{i \pi(k+i x)}}{a^{k+i x}} \Gamma(k+i x)+\frac{e^{-i \pi(k-i x)}}{a^{k-i x}} \Gamma(k-i x)\right\} d x=e^{a} .
$$

Entry 1.3. If $a>0,0 \leq \lambda<\epsilon$, and $1 / \epsilon$ is a positive integer, then

$$
\epsilon \sum_{n=0}^{\infty} \frac{a^{\lambda+n \epsilon}}{\Gamma(1+\lambda+n \epsilon)}=e^{a}-\frac{\epsilon}{\pi} \int_{0}^{\infty} e^{-a x} x^{-\lambda-1} \frac{\sin \pi(\lambda-\epsilon)-x^{\epsilon} \sin \pi \lambda}{2 \cos \pi \epsilon-\left(x^{\epsilon}+x^{-\epsilon}\right)} d x .
$$

In the next section, we prove Theorems 1.1 and 1.2 while in the last section we discuss Entry 1.3. We demonstrate its incorrectness by showing that as $a \rightarrow \infty$, the two sides of (1.5) have different asymptotic expansions. On the other hand, if we regard the left side of (1.5) as a Riemann sum, then the limits of both sides as $\epsilon \rightarrow 0$ are equal.

\section{Proofs of Theorems 1.1 and 1.2}

Proof of Theorem [1.1. Let $f(a, k)$ denote the left side of (1.3). Then, by straightforward differentiation, the reflection formula for $\Gamma(k)$, and the standard integral representation for $\Gamma(k)$,

$$
\begin{aligned}
\frac{\partial}{\partial k} f(a, k)= & \frac{a^{-k}}{\Gamma(1-k)}+\int_{0}^{\infty} \frac{e^{-a x} x^{k-1} \log x}{\pi^{2}+\log ^{2} x}\left(\cos \pi k-\frac{1}{\pi} \sin \pi k \log x\right) d x \\
& +\int_{0}^{\infty} \frac{e^{-a x} x^{k-1}}{\pi^{2}+\log ^{2} x}(-\pi \sin \pi k-\cos \pi k \log x) d x \\
= & \frac{a^{-k}}{\Gamma(1-k)}-\frac{\sin \pi k}{\pi} \int_{0}^{\infty} e^{-a x} x^{k-1} d x \\
= & \frac{a^{-k}}{\pi} \Gamma(k) \sin \pi k-\frac{\sin \pi k}{\pi} a^{-k} \Gamma(k)=0 .
\end{aligned}
$$

Hence, $f(a, k)$ is constant with respect to $k$.

Next, differentiating with respect to $a$ and using the functional equation for the $\Gamma$-function, we find that

$$
\begin{aligned}
\frac{\partial}{\partial a} f(a, k) & =\int_{-k}^{\infty} \frac{x a^{x-1}}{\Gamma(x+1)} d x-\int_{0}^{\infty} \frac{e^{-a x} x^{k}}{\pi^{2}+\log ^{2} x}\left(\cos \pi k-\frac{1}{\pi} \sin \pi k \log x\right) d x \\
& =\int_{-k-1}^{\infty} \frac{a^{x}}{\Gamma(x+1)} d x+\int_{0}^{\infty} \frac{e^{-a x} x^{k}}{\pi^{2}+\log ^{2} x}\left(\cos \pi(k+1)-\frac{1}{\pi} \sin \pi(k+1) \log x\right) d x \\
(2.2) \quad & =f(a, k+1) .
\end{aligned}
$$

Thus, by (2.1) and (2.2),

$$
\frac{\partial f}{\partial a}(a, k)=f(a, k)
$$

It follows that, for some constant $c$,

$$
f(a, k)=c e^{a} .
$$

It remains to evaluate $c$, and, more precisely, in order to prove (1.3), we must show that $c=1$. 
To prove that $c=1$, we evaluate $f(a, k)$ when $a=k=0$. From the definition of $f$ and the substitution $u=\log x$, we see that

$$
f(0,0)=\int_{0}^{\infty} \frac{d x}{x\left(\pi^{2}+\log ^{2} x\right)}=\int_{-\infty}^{\infty} \frac{d u}{\pi^{2}+u^{2}}=1 .
$$

Using (2.4) in (2.3), we conclude that $c=1$, as desired.

Proof of Theorem 1.2. Let $f(a, k)$ denote the left side of (1.4). Then,

$$
\begin{aligned}
\frac{\partial}{\partial k} f(a, k)= & \frac{a^{-k}}{\Gamma(1-k)}+\frac{i \pi}{2 \pi} \int_{0}^{\infty}\left\{\frac{e^{i \pi(k+i x)}}{a^{k+i x}} \Gamma(k+i x)-\frac{e^{-i \pi(k-i x)}}{a^{k-i x}} \Gamma(k-i x)\right\} d x \\
& -\frac{\log a}{2 \pi} \int_{0}^{\infty}\left\{\frac{e^{i \pi(k+i x)}}{a^{k+i x}} \Gamma(k+i x)+\frac{e^{-i \pi(k-i x)}}{a^{k-i x}} \Gamma(k-i x)\right\} d x \\
& +\frac{1}{2 \pi} \int_{0}^{\infty}\left\{\frac{e^{i \pi(k+i x)}}{a^{k+i x}} \frac{\partial}{\partial k} \Gamma(k+i x)+\frac{e^{-i \pi(k-i x)}}{a^{k-i x}} \frac{\partial}{\partial k} \Gamma(k-i x)\right\} d x .
\end{aligned}
$$

Now by the chain rule,

$$
\frac{\partial}{\partial k} \Gamma(k \pm i x)=\mp i \frac{\partial}{\partial x} \Gamma(k \pm i x) .
$$

Hence, using (2.6) in (2.5) and integrating by parts, we find that

$$
\begin{aligned}
\frac{\partial}{\partial k} f(a, k)= & \frac{a^{-k}}{\Gamma(1-k)}+\frac{i}{2} \int_{0}^{\infty}\left\{\frac{e^{i \pi(k+i x)}}{a^{k+i x}} \Gamma(k+i x)-\frac{e^{-i \pi(k-i x)}}{a^{k-i x}} \Gamma(k-i x)\right\} d x \\
& -\frac{\log a}{2 \pi} \int_{0}^{\infty}\left\{\frac{e^{i \pi(k+i x)}}{a^{k+i x}} \Gamma(k+i x)+\frac{e^{-i \pi(k-i x)}}{a^{k-i x}} \Gamma(k-i x)\right\} d x \\
& +\frac{1}{2 \pi i}\left\{\left.\frac{e^{i \pi(k+i x)}}{a^{k+i x}} \Gamma(k+i x)\right|_{0} ^{\infty}-\left.\frac{e^{-i \pi(k-i x)}}{a^{k-i x}} \Gamma(k-i x)\right|_{0} ^{\infty}\right\} \\
& -\frac{1}{2 \pi i} \int_{0}^{\infty}\left\{\frac{-\pi e^{i \pi(k+i x)}}{a^{k+i x}} \Gamma(k+i x)+\frac{\pi e^{-i \pi(k-i x)}}{a^{k-i x}} \Gamma(k-i x)\right\} d x \\
& +\frac{i \log a}{2 \pi i} \int_{0}^{\infty}\left\{\frac{e^{i \pi(k+i x)}}{a^{k+i x}} \Gamma(k+i x)+\frac{e^{-i \pi(k-i x)}}{a^{k-i x}} \Gamma(k-i x)\right\} d x .
\end{aligned}
$$

Observe that, by Stirling's formula for the $\Gamma$-function on a vertical line [6, p. 945], the expressions for the integrated terms vanish at $\infty$. After considerable cancellation, (2.7) reduces simply to

$$
\begin{aligned}
\frac{\partial}{\partial k} f(a, k) & =\frac{a^{-k}}{\Gamma(1-k)}-\frac{1}{2 \pi i}\left(\frac{e^{i \pi k}}{a^{k}}-\frac{e^{-i \pi k}}{a^{k}}\right) \Gamma(k) \\
& =\frac{a^{-k}}{\pi} \sin (\pi k) \Gamma(k)-\frac{a^{-k}}{\pi} \sin (\pi k) \Gamma(k)=0,
\end{aligned}
$$

upon again using the reflection formula for the $\Gamma$-function. Thus, $f(a, k)$ is constant with respect to $k$. 
Next, differentiating and using the functional equation of the $\Gamma$-function, we find that

$$
\begin{aligned}
\frac{\partial}{\partial a} f(a, k)= & \int_{-k-1}^{\infty} \frac{a^{x}}{\Gamma(x+1)} d x+\frac{1}{2 \pi} \int_{0}^{\infty}\left\{\frac{e^{i \pi(k+1+i x)}}{a^{k+1+i x}} \Gamma(k+1+i x)\right. \\
& \left.+\frac{e^{-i \pi(k+1-i x)}}{a^{k+1-i x}} \Gamma(k+1-i x)\right\} d x \\
= & f(a, k+1) .
\end{aligned}
$$

Hence, by (2.8),

$$
\frac{\partial}{\partial a} f(a, k)=f(a, k)
$$

and so, for some constant $c$,

$$
f(a, k)=c e^{a} .
$$

By (1.4), it remains to show that $c=1$.

To determine $c$, we evaluate $f(a, k)$ when $a=1$ and $k=0$. Recalling that $f(a, k)$ denotes the left side of (1.4), we see that

$$
f(1,0)=\int_{0}^{\infty} \frac{d x}{\Gamma(x+1)}+\frac{1}{2 \pi} \int_{0}^{\infty}\left\{e^{-\pi x}(\Gamma(i x)+\Gamma(-i x))\right\} d x .
$$

To evaluate the latter integral, examine, for $\epsilon>0$,

$$
I_{\epsilon}:=\frac{1}{2 \pi} \int_{0}^{\infty}\left\{e^{-\pi x}(\Gamma(\epsilon+i x)+\Gamma(\epsilon-i x))\right\} d x .
$$

Inserting the integral representations for $\Gamma(\epsilon \pm i x)$ in (2.12) and inverting the order of integration by absolute convergence, we find that

$$
\begin{aligned}
I_{\epsilon} & =\frac{1}{2 \pi}\left(\int_{0}^{\infty} \frac{e^{-t}}{t^{1-\epsilon}} d t \int_{0}^{\infty} e^{-x(\pi-i \log t)} d x+\int_{0}^{\infty} \frac{e^{-t}}{t^{1-\epsilon}} d t \int_{0}^{\infty} e^{-x(\pi+i \log t)} d x\right) \\
& =\frac{1}{2 \pi} \int_{0}^{\infty} \frac{e^{-t}}{t^{1-\epsilon}}\left(\frac{1}{\pi-i \log t}+\frac{1}{\pi+i \log t}\right) d t \\
& =\int_{0}^{\infty} \frac{e^{-t}}{t^{1-\epsilon}\left(\pi^{2}+\log ^{2} t\right)} d t .
\end{aligned}
$$

Since

$$
\int_{0}^{\infty} \frac{e^{-t}}{t\left(\pi^{2}+\log ^{2} t\right)} d t<\infty
$$

by the Lebesgue dominated convergence theorem, we may take the limit as $\epsilon \rightarrow 0$ under the integral sign on the right side of (2.13). Thus, from (2.13) and (2.11),

$$
f(1,0)=\int_{0}^{\infty} \frac{d t}{\Gamma(t+1)}+\int_{0}^{\infty} \frac{e^{-t}}{t\left(\pi^{2}+\log ^{2} t\right)} d t=e
$$

by (1.3). Hence, by (2.10), $c=1$, as desired. 


\section{Discussion of Entry 1.3}

We first observe that the sum on the left side of (1.5) is a Riemann sum in which each subinterval is of length $\epsilon$, and the function $a^{x} / \Gamma(1+x)$ is evaluated at the point $\lambda+n \epsilon$ in the $n$th subinterval, where $n \geq 0$ and $0 \leq \lambda<\epsilon$. Thus, as $\epsilon \rightarrow 0$, the left side of (1.5) tends to the first integral on the left side of (1.3) when $k=0$. For the integral on the right side of (1.5), we can let $\epsilon \rightarrow 0$ inside the integral sign by the dominated convergence theorem. Recalling that $0 \leq \lambda<\epsilon$, we will assume that $\lambda$ is a twice differentiable function of $\epsilon$ in applying L'Hospital's rule. After a straightforward but not so short calculation, we find that

$$
\lim _{\epsilon \rightarrow 0} \frac{\epsilon\left(\sin \pi(\lambda-\epsilon)-x^{\epsilon} \sin \pi \lambda\right)}{2 \cos \pi \epsilon-\left(x^{\epsilon}+x^{-\epsilon}\right)}=\frac{\pi}{\pi^{2}+\log ^{2} x} .
$$

Thus, letting $\epsilon \rightarrow 0$ on both sides of (1.5), we find that the proposed equality becomes

$$
\int_{0}^{\infty} \frac{a^{x}}{\Gamma(x+1)} d x=e^{a}-\int_{0}^{\infty} \frac{e^{-a x}}{x\left(\pi^{2}+\log ^{2} x\right)} d x
$$

which is true, by (1.3) with $k=0$, and so Entry 1.3 is valid in the limit as $\epsilon \rightarrow 0$.

When $\lambda=0$ and $\epsilon=1$, then both sides of (1.5) are equal to $e^{a}$. Numerical calculations also show that for $\lambda=\frac{1}{2}, \epsilon=1$, and small $a$, e.g., $a=1,2$, the two sides of (1.5) agree to at least 30 decimal places.

We now show that (1.5) is not valid in general. For simplicity, we choose $\lambda=\frac{1}{2}$ and $\epsilon=1$ and show asymptotically that as $a \rightarrow \infty$, the left and right sides of (1.5) have different asymptotic expansions. Similar arguments are valid for other fixed values of $\lambda$ and $\epsilon$. Now

$$
\frac{1}{\sqrt{x}(1+x)}=\sum_{n=0}^{\infty}(-1)^{n} x^{n-1 / 2}, \quad 0<x<1,
$$

and so by a routine application of Watson's Lemma [8, p. 113], the right side of (1.5) has the asymptotic expansion, as $a \rightarrow \infty$,

$$
e^{a}-\frac{1}{\pi} \sum_{n=0}^{\infty} \frac{(-1)^{n} \Gamma\left(n+\frac{1}{2}\right)}{a^{n+1 / 2}} .
$$

On the left side of (1.5), we use a result of Ramanujan from Chapter 3 in his second notebook 9], 2, pp. 57, 58, Entry 10] along with the familiar asymptotic expansion [1, p. 257, formula 6.147]

$$
x^{b-a} \frac{\Gamma(x+a)}{\Gamma(x+b)}=1+\frac{(a-b)(a+b-1)}{2 x}+O\left(\frac{1}{x^{2}}\right)
$$

as $x \rightarrow \infty$. A complete statement of Entry 10 in Chapter 3 is too long to give here, but it suffices to say that we are applying Entry 10 to $\phi(x)=\Gamma(x+1) / \Gamma\left(x+\frac{3}{2}\right)$, which easily satisfies the theorem's hypotheses. Accordingly, we find from (3.2) 
that, as $a \rightarrow \infty$,

$$
\begin{aligned}
\sum_{n=0}^{\infty} \frac{a^{n+1 / 2}}{\Gamma\left(n+\frac{3}{2}\right)} & =\sqrt{a} \sum_{n=0}^{\infty} \frac{a^{n}}{n !} \frac{\Gamma(n+1)}{\Gamma\left(n+\frac{3}{2}\right)} \\
& =e^{a} \sqrt{a}\left\{\frac{\Gamma(a+1)}{\Gamma\left(a+\frac{3}{2}\right)}+O\left(\frac{1}{a^{5 / 2}}\right)\right\} \\
& =e^{a} \sqrt{a}\left\{a^{-1 / 2}\left(1-\frac{3}{8 a}+O\left(\frac{1}{a^{2}}\right)\right)+O\left(\frac{1}{a^{5 / 2}}\right)\right\} \\
& =e^{a}\left\{1-\frac{3}{8 a}+O\left(\frac{1}{a^{2}}\right)\right\} .
\end{aligned}
$$

A comparison of (3.3) with (3.1) shows that the left and right sides of (1.5) have different asymptotic expansions as $a \rightarrow \infty$, and so (1.5) cannot be true in general. In conclusion, however, we remark that in his notebooks, Ramanujan often wrote equality signs for asymptotic expansions and approximations; for example, he never used the symbols $\sim$ or $\approx$. Thus, it is most likely that Ramanujan himself did not regard (1.5) as an equality. 3

The author thanks Jonathan Borwein for a significant simplification in Section

\section{REFERENCES}

[1] M. Abramowitz and I. A. Stegun, Handbook of Mathematical Functions, Dover, New York, 1965. MR 34:8606

[2] B. C. Berndt, Ramanujan's Notebooks, Part I, Springer-Verlag, New York, 1985. MR 86c:01062

[3] B. C. Berndt, Ramanujan's Notebooks, Part II, Springer-Verlag, New York, 1989. MR 90b:01039

[4] R. P. Boas and H. Pollard, Continuous analogues of series, Amer. Math. Monthly 80 (1973), 18-25. MR 47:3903

[5] P. J. Forrester, Extensions of several summation formulae of Ramanujan using the calculus of residues, Rocky Mt. J. Math. 13 (1983), 557-572. MR 85i:40004

[6] I. S. Gradshteyn and I. M. Ryzhik, Table of Integrals, Series, and Products, 5th ed., Academic Press, San Diego, 1994. MR 94g:00008

[7] K. S. Krishnan, On the equivalence of certain infinite series and the corresponding integrals, J. Indian Math. Soc. 12 (1948), 79-88. MR 10:530d

[8] F. W. J. Olver, Asymptotics and Special Functions, Academic Press, New York, 1974. MR 55:8655

[9] S. Ramanujan, Notebooks (2 volumes), Tata Institute of Fundamental Research, Bombay, 1957. MR 20:6340

[10] S. Ramanujan, The Lost Notebook and Other Unpublished Papers, Narosa, New Delhi, 1988. MR 89j:01078

Department of Mathematics, University of Illinois, 1409 West Green Street, UrBANA, ILLINOIS 61801

E-mail address: berndt@math.uiuc.edu 